Journal of Case Reports 2020;10(2):103-106

\title{
An Organic Cause for Perceived Muscle Weakness
}

\author{
Thangavelu Senthilvelkumar ${ }^{1}$, Thamaraiselvan Aravindan ${ }^{1}$, Ajith Sivadasan ${ }^{2}$ \\ ${ }^{1}$ Department of Physical Medicine and Rehabilitation; ${ }^{2}$ Department of Neurology and Neurosciences, Christian Medical College, \\ Vellore 632004, Tamil Nadu, India.
}

\section{Corresponding Author:}

Mr. Thangavelu Senthilvelkumar

Email: sentheel@gmail.com

This is an Open Access article distributed under the terms of the Creative Commons Attribution License (creativecommons.org/ licenses/by/3.0).

Received Accepted

Published

March 29, 2020

May 9, 2020

May 20, 2020

\begin{abstract}
Background: Although neurological conditions present with specific clinical patterns, practitioners often encounter patients with vague symptom complexes. Delayed diagnosis and unnecessary medications can lead to iatrogenic effects and poor prognosis. Case Report: This paper reports a 21-year-old girl who perceived right upper extremity monoplegia following a trauma, which did not fit into the traditional neurological diagnosis. Her movement patterns during physiotherapy were suggestive of the central fatigue related reduction of function. Focused behavioral neuro-rehabilitation approach based training helped her to regain her upper extremity function. Conclusion: Central fatigue may be considered as a differential diagnosis when atypical muscle weakness occurs following a probable physical exertion.
\end{abstract}

Keywords: Differential Diagnosis, Fatigue, Muscle Weakness, Nervous System Diseases, Prognosis.

\section{Introduction}

Although neurological conditions present with specific clinical patterns, practitioners often encounter patients with vague symptom complexes. With few exceptions, motor weakness fits into either an upper motor neuron or lower motor neuron pattern with a definitive organic cause. Neurological symptoms unexplained by other traditional neurological or medical conditions are classified as functional neurological symptom disorder [1]. These symptoms are usually nonorganic in origin. When patients present with atypical symptoms diagnosis becomes a challenge. Here, we report a 21-year-old girl who perceived right upper extremity monoplegia following a trauma which did not fit into the traditional neurological diagnosis.

\section{Case Report}

A 21 year girl perceived right upper limb weakness after carrying a pot of half-filled water for a 100 meter distance. She expressed that while carrying the pot, the maximum force was exerted on her ring and middle finger. At the destination, she literally dropped the pot and experienced severe pain at right metacarpo-phalangeal joint of ring and middle finger. Immediately after which she felt numbness below the elbow and was unable to move the entire upper extremity. She was taken to a nearby orthopedic surgeon who suspected her to have had a wrist injury. She was hence put on posterior slab and arm sling for a week's time. To identify the cause for her weakness, cervical MRI was done and there was no abnormality. Subsequently, she was referred to our institution for further evaluation and management. She underwent a series evaluation by spine surgeon, hand surgeon and a neurologist. The sensory function, muscle tone, reflexes showed no abnormality. The possibility of a non-organic cause for the symptoms was considered. Normal electrophysiological studies supported the clinical diagnosis. 
The neurologist referred her for physiotherapy to improve the right upper extremity function. At that time, she started making little movement in her finger. During physiotherapy evaluation, she had muscle power of 1 out of 5 in shoulder and elbow as measured by manual muscle testing. There was a little finger movement. Her Motricity index score for upper extremity was 39 out of 100 and the upper extremity functional index (UEFI) score was 5 out of 80 . She also had shoulder pain with the score of 6 in visual analogue scale (VAS). The therapy focused to improve her upper extremity weakness and shoulder pain. Since the presentation was atypical, the search of non-organic reasons for her weakness other than usual ones continued. During the therapy we found that her spontaneous activities were better than volitional activities. This was evident during the application of moist heat pack for her shoulder pain. She spontaneously pulled the pack with her right hand as she felt increased heat. During this incident, she demonstrated movements much higher than her available voluntary control.

This incident made us to think differently because forced movements are better in quantity. So, we introduced constraint induced movement therapy (CIMT) to her. It is a behavioral neurorehabilitation approach based on 'Learned Nonuse' [2-4]. This concept is originally developed by Dr Edward Taub. We restrained her left hand and forced her to use her right hand for all the ADL activities. She was put on intensive repetitive structured movements in the right affected arm. She was asked to actively use her affected arm 6-7 hours per day for 7 days. After 7 days of CIMT training, her right upper extremity improved drastically. Her Motricity index score increased to 88 out of 100 and her UEFI score increased to 61 from the initial score of 5. She was able to perform all her ADL activities and started writing using her right extremity. She was advised to continue progressive muscle strengthening program and sent back to continue her education.

\section{Discussion}

We presented a unique case atypical upper extremity weakness which did not fall into conventional diagnostic criteria. Though the nonorganic features are considered, it was noted that the key features of non-organic weakness like inconsistency and distractibility were not present [5]. Although her weakness was abrupt, it was after an acute injury. This weakness was not associated with any sensory abnormality and distractibility. Today, central fatigue is commonly accepted as a physiological phenomenon that plays a relevant role in muscle fatigue that protects muscles against excessive effort [6,7]. Muscle fatigue is a protective mechanism of body that prevents further damage of muscles. Central nervous system fatigue is a key component in preventing peripheral muscle injury [8]. The brain can reduce the quantity of motor commands sent from the central nervous system. It can also be considered as a state of exhaustion. We believe that mental fatigue or central fatigue could have been a reason for her perceived weakness.

The reduction of motor commands sent from the brain increases the amount of perceived effort an individual experiences By forcing the body through a higher perceived intensity, the individual becomes more likely to cease exercise by means of exhaustion. Perceived effort is greatly influenced by the intensity of corollary discharge from the motor cortex that affects the primary somatosensory cortex [9]. Noakes and others [9-11] have developed the "central governor" model of central fatigue that focuses primarily on the conscious and subconscious brain and does not involve the spinal cord or motor unit. This phenomenon is well explained for exercise related fatigue. But in our case, it occurred following a static sustained muscle work. The central fatigue model posits that the reduction in power output during prolonged exercise leading to the cessation of exercise is not caused by limiting physiological processes in any of the peripheral organs including 
the active skeletal muscles. Rather, the fatigue related reductions in skeletal muscle power output are caused by altered efferent command from the brain [12].

In this reported case, the motor command to the entire right upper extremity was inhibited in order to reduce further damage. The time course between central fatigue and recovery is always short. But in this case, it was extended for weeks. Other researches confirm that CNS fatigue is evident directly on post-workout; even though muscle soreness and peripheral neuromuscular fatigue took over 3 days to recover [13].

The central and peripheral fatigues may have worked together to minimize the right upper extremity activity. Latella et al. [14] studied the time-course of CNS recovery after strength training. They managed to induce a whopping $46 \%$ decrease in corticospinal excitability (measured by motorevoked potential). This means major CNS fatigue. It took 20 minutes for the CNS to recover [14]. But it is difficult to provide theoretical explanation for the 100 percentage reduction in the motor output occurred. A better way to prove the CNS fatigue is through motor-evoked potential (MEP) $[15,16]$. Similar case situations in future may warrant MEP to confirm reduction in corticospinal excitability.

A common trap in this type of case scenario is to assume that the condition is non-organic in origin, thereby possibly missing an important diagnosis. Delayed diagnosis and unnecessary medications can lead to iatrogenic effects, delay appropriate treatment and negatively affect prognosis $[17,18]$.

\section{Conclusion}

Central fatigue may be considered as a differential diagnosis when atypical muscle weakness occurs following a probable physical exertion.

Contributors: TS drafted the manuscript, did literature search and patient management; TA and AS edited the manuscript and were involved in patient management. TS will act as a study guarantor. All authors approved the final version of the manuscript and are responsible for all aspects of the study.

Funding: None; Competing interests: None stated.

\section{References}

1. American Psychiatric Association. Diagnostic and Statistical Manual of Mental Disorders [Internet]. Fifth Edition. American Psychiatric Association; 2013 [cited 2019 Jul 28].

2. Taub E, Uswatt G. Constraint-induced movement therapy: answers and questions after two decades of research. Neuro Rehabilitation. 2006;21:93-95.

3. Nadeau SE, Wu SS. CIMT as a behavioral engine in research on physiological adjuvants to neurorehabilitation: the challenge of merging animal and human research. Neuro Rehabilitation. 2006;21:107130.

4. Taub E, Uswatte G, Mark VW, Morris DMM. The learned nonuse phenomenon: implications for rehabilitation. Eura Medicophys. 2006;42:241-256.

5. Williams DT, Ford B, Fahn S. Phenomenology and psychopathology related to psychogenic movement disorders. Adv Neurol. 1995;65:231-257.

6. Bigland-Ritchie B, Johansson R, Lippold OC, Woods JJ. Contractile speed and EMG changes during fatigue of sustained maximal voluntary contractions. J Neurophysiol. 1983;50:313-324.

7. Gandevia SC. Spinal and supraspinal factors in human muscle fatigue. Physiol Rev. 2001;81:1725-1789.

8. Noakes TD. Fatigue is a brain-derived emotion that regulates the exercise behavior to ensure the rrotection of whole body homeostasis. Front Physiol. 2012;3:82.

9. Enoka RM, Stuart DG. Neurobiology of muscle fatigue. J Appl Physiol. 1992;72:1631-1648.

10. Lambert E, St C, Noakes T. Complex systems model of fatigue: integrative homoeostatic control of peripheral physiological systems during exercise in humans. Br J Sports Med. 2005;39:52-62.

11. Noakes TD, St Clair Gibson A. Logical limitations to the "catastrophe" models of fatigue during exercise in humans. Br J Sports Med. 2004;38:648-649.

12. Gandevia SC. Neural control in human muscle fatigue: changes in muscle afferents, motoneurones and motor cortical drive [corrected]. Acta Physiol Scand. 1998; 162:275-283.

13. Behrens M, Mau-Moeller A, Bruhn S. Effect of exerciseinduced muscle damage on neuromuscular function of the quadriceps muscle. Int J Sports Med. 2012;33:600606.

14. Latella C, Hendy AM, Pearce AJ, Vander Westhuizen D, Teo WP. The time-course of acute changes in corticospinal excitability, intra-cortical inhibition and facilitation following a single-session heavy strength 
training of the biceps brachii. Front Hum Neurosci [Internet]. 2016 Dec 1;10. Available from: https://www. ncbi.nlm.nih.gov/pmc/articles/PMC5133051/ Accessed on March 29, 2020.

15. Smith JL, Martin PG, Gandevia SC, Taylor JL. Sustained contraction at very low forces produces prominent supraspinal fatigue in human elbow flexor muscles. J Appl Physiol. 2007;103:560-568.

16. Teo WP, Rodrigues JP, Mastaglia FL, Thickbroom GW. Post-exercise depression in corticomotor excitability after dynamic movement: a general property of fatiguing and non-fatiguing exercise. Exp Brain Res. 2012;216:4149.

17. Reuber M. The etiology of psychogenic non-epileptic seizures: Toward a biopsychosocial model. Neurologic Clinics. 2009;27:909-924.

18. Fobian AD, Elliott L. A review of functional neurological symptom disorder etiology and the integrated etiological summary model. J Psychiatry Neurosci. 2019;44:8-18. 